\title{
What did the 2010s ever do for us?
}

\author{
Tom Moberly UK editor
}

The BMJ

The past decade has been characterised largely by the fallout of the global financial crash, a revolution in the way we use technology to buy goods and interact with one another, and the return of populism and nationalism as potent political forces. While these have affected all our lives, including our healthcare, they aren't the only issues that have shaped the decade.

The Ebola and Zika outbreaks have reminded us of the continuing threat that infectious diseases pose in an era of global mass transit (doi:10.1136/bmj.16894). In the UK the publication of Robert Francis's report on care at Mid Staffordshire NHS Foundation Trust in February 2010 led to a renewed focus on patient safety. And disputes between doctors and the government over pensions in 2012 and the junior doctor contract in 2015 prompted industrial action and led to wider discussions about pay, conditions, and working culture and wellbeing among doctors.

Nigel Hawkes believes that for the UK it has overall been a "decade of disappointment" characterised by faltering progress in healthcare outcomes (doi:10.1136/bmj.16895). "Decades of improvement in life expectancy have plateaued, even gone into reverse," he writes. "Neonatal health has paused in its steady improvement. Doctors are demoralised, GP surgeries and hospitals are under siege, and public satisfaction with the NHS, which rose sharply between 2000 and 2010, has fallen back as quickly as it rose."
Others are less pessimistic (doi:10.1136/bmj.16889). Karishma Singh welcomes the way UK general practice has adapted to meet patients' rising expectations and demand. Emma Plunkett believes there is now more awareness of the importance of doctors looking after their own wellbeing. And Johann Malawana sees hope for the future in the passion and vision of young UK clinicians.

Meanwhile, Rebecca Coombes looks at how the trial misconduct, hidden research findings, and conflicts of interest that The BMJ has uncovered have led to changes in guidelines and practice and to parliamentary inquiries in the UK and European Union (doi:10.1136/bmj.16892). And Elizabeth Loder's selection of the "finest" $B M J$ research papers of the decade reminds us that screening healthy people is not always without risk and that exercise, for many conditions, is as good as drugs (doi:10.1136/bmj.16888).

The 2010s have also been marked by growing efforts to base healthcare decisions on trustworthy evidence. Ray Moynihan and colleagues point out that it is now a decade since the US Institute of Medicine's landmark report highlighted the problem of financial conflicts of interest undermining scientific inquiry, medical education, and patient care. In their Analysis article (doi:10.1136/bmj.16576), they set out a route by which they hope the healthcare sector can reach a situation where decision making is financially independent from industry. Let us hope we are able to report on meaningful progress down this road before the next decade is out. 